\title{
UNE NOUVELLE FILAIRE, CHABFILARIA JONATHANI n. gen., n. sp., Onchocercidae parasite de Xénarthre ${ }^{1}$
}

\author{
O. BAIN*, PURNOMO** et J. P. DEDET***
}

\begin{abstract}
RÉSUMÉ. Description de Chabfilaria jonathani n. gen., n. sp., parasite de Choloepus didactylus, Bradypodidae de Guyane Française. Cette espèce, ainsi que Chabfilaria freitaslenti (Yeh, 1957) n. comb., parasite de Myrmecophaga tridactyla, constituent un genre intéressant par ses caractères primitifs, qui pourrait représenter un lien entre les Onchocercinae et les Setariinae.

On connaît maintenant 11 espèces de Filaires parasites de Xénarthes ; ce sont des représentants primitifs des Onchocercinae, des Dirofilariinae et, semble-t-il avec Chabfilaria, des Setariinae, donc des 3 grands groupes actuellement diversifiés chez les Mammifères. La région néotropicale, avec sa faune primitive (Marsupiaux, Xénarthres, Protongulés) a donc peut-être une importance particulière dans l'origine des Filaires de Mammifères.
\end{abstract}

\section{A new Filaria, Chabfilaria jonathani n. gen., n. sp., Onchocercidae parasite of Xenarthra.}

SUMMARY. Chafilaria jonathani n. gen., n. sp. is described from Choloepus didactylus (Family Bradypodidae) from French Guyana. This species and C. freitaslenti (Yeh, 1957) n. comb., constitute a genus interesting because of its primitive characters, which could represent a link between the Onchocercinae and the Setariinae.

Eleven species of filaria are known from the Xenarthra. They are primitive members of the Onchocercinae, the Dirofilariinae and if our notions on Chabfilaria are correct, the Setariinae; thus, the three major groups which occur in mammals. The neotropical region with its primitive fauna (Marsupials, Xenarthra and Protoungulates) may be of particular importance in understanding the origins of the filaria of mammals.

I. Ce travail a pu être effectué grâce à une subvention de l'Organisation Mondiale de la Santé et grâce à l'aide du Ministère de la Recherche et de la Technologie à l'Institut Pasteur de Cayenne (Programme Épidémiologie de la leishmamiose en Guyane).

* Laboratoire de Zoologie des Vers, associé au CNRS, Muséum National d'Histoire Naturelle, 61, rue Buffon, F 75231 Paris Cedex 05. Indonesia.

** Department of Parasitology, Faculty of Medicine, University of Indonesia, Salemba 4, Jakarta,

*** Institut Pasteur de la Guyane Française, B. P. 304, 97305 Cayenne Cedex, Guyane Française. Accepté le 23 février 1983 . 
Les Filaires que nous décrivons ont été récoltées chez un Paresseux, Choloepus didactylus L., provenant de Cacao, en Guyane Française. Elles sont situées sous le plan musculaire externe de la panse et ont des microfilaires sanguines.

Le matériel comprend 1 ㅇ holotype, $1 \hat{\delta}$ allotype, 1 \& et $2 \hat{\jmath}$ paratypes (lot $677 \mathrm{NE}$, Muséum National d'Histoire Naturelle, Paris).

\section{Description}

Cuticule épaisse ; couche externe avec des rangées de petits bâtonnets longitudinaux régulièrement disposés entre les stries transversales du corps ( fig. $1 \mathrm{D}$ et $3 \mathrm{~F}$ ) ; couche interne à fibres obliques entrecroisées. Pas d'ailes latérales saillantes sur le corps mais deux épaississements cuticulaires internes latéraux (fig. 1 C).

Région antérieure brusquement amincie, de l'anneau nerveux à l'apex ( $f i g .1 \mathrm{~A}$ et 2 A). Deirides bifides.

Nombre et disposition des papilles de la tête indiqués sur les figures $1 \mathrm{~K}, L$ et $M$; deux volumineuses papilles labiales externes latérales sont présentes ainsi que 2 légers reliefs céphaliques latéraux situés entre ces deux papilles et la bouche (ils ne sont bien visibles qu'en vue médiane, fig. $1 \mathrm{~L}$ ). Bouche triangulaire - arrondie ; cavité buccale à section transversale plus ou moins ovale ; capsule buccale de deux segments (fig. $1 \mathrm{~N}$ ), l'antérieur orné de rides longitudinales sur la face interne ( fig. 10 ). Esophage très long, épais, à lumière en $\mathrm{Y}$ en section transversale ; portion musculaire avec un segment antérieur court et un segment postérieur plus long, distincts l'un de l'autre par un changement d'aspect de la lumière œsophagienne, un peu en avant de l'anneau nerveux ( fig. $1 \mathrm{~A}$ et $2 \mathrm{C}$ ) ; portion glandulaire très longue (environ $1 \mathrm{~cm}$ ). Intestin à paroi épaisse ( fig. $1 \mathrm{~B}$ ).

Queue conique, phasmides subterminales; 0,1 ou 2 pointes cuticulaires coniques ou tronquées, latérales et subterminales ( fig. $1 \mathrm{~J}, 2 \mathrm{H}, \mathrm{I}$ et $\mathrm{J}$ ).

\section{Femelle (fig. 1).}

Vulve au niveau de l'œsophage musculaire ; vagin complexe à vagina vera court et sphincter formé par un tube coudé étroit entouré d'une puissante musculature et fermé en avant par une valvule de 4 (?) cellules épithéliales saillantes ( fig. $1 \mathrm{E}$ ). Fusion des deux utérus à $2 \mathrm{~mm}$ de la vulve. Opisthodelphie. Spermatozoïdes entassés à la partie distale des utérus ( $f$ ig. $1 \mathrm{~F}$ ).

Queue courbée dorsalement et légèrement spiralée autour de l'axe longitudinal. Dimensions : + holotype : corps long de $73 \mathrm{~mm}$ et large de $300 \mu \mathrm{m}$; anneau nerveux et deirides à 110 et $430 \mu \mathrm{m}$ de l'apex; œsophage long de $13.320 \mu \mathrm{m}$ avec portion musculaire longue de $670 \mu \mathrm{m}$; vulve à $590 \mu \mathrm{m}$ de l'apex ; portion impaire de l'ovéjecteur longue de $2.250 \mu \mathrm{m}$; queue longue de $320 \mu \mathrm{m}$.

Autre + : corps long de $76 \mathrm{~mm}$ et large de $410 \mu \mathrm{m}$; anneau nerveux, deirides et cellule excrétrice à $120 \mu \mathrm{m}, 500 \mu \mathrm{m}$ et $400 \mu \mathrm{m}$ de l'apex ; cavité buccale haute de 

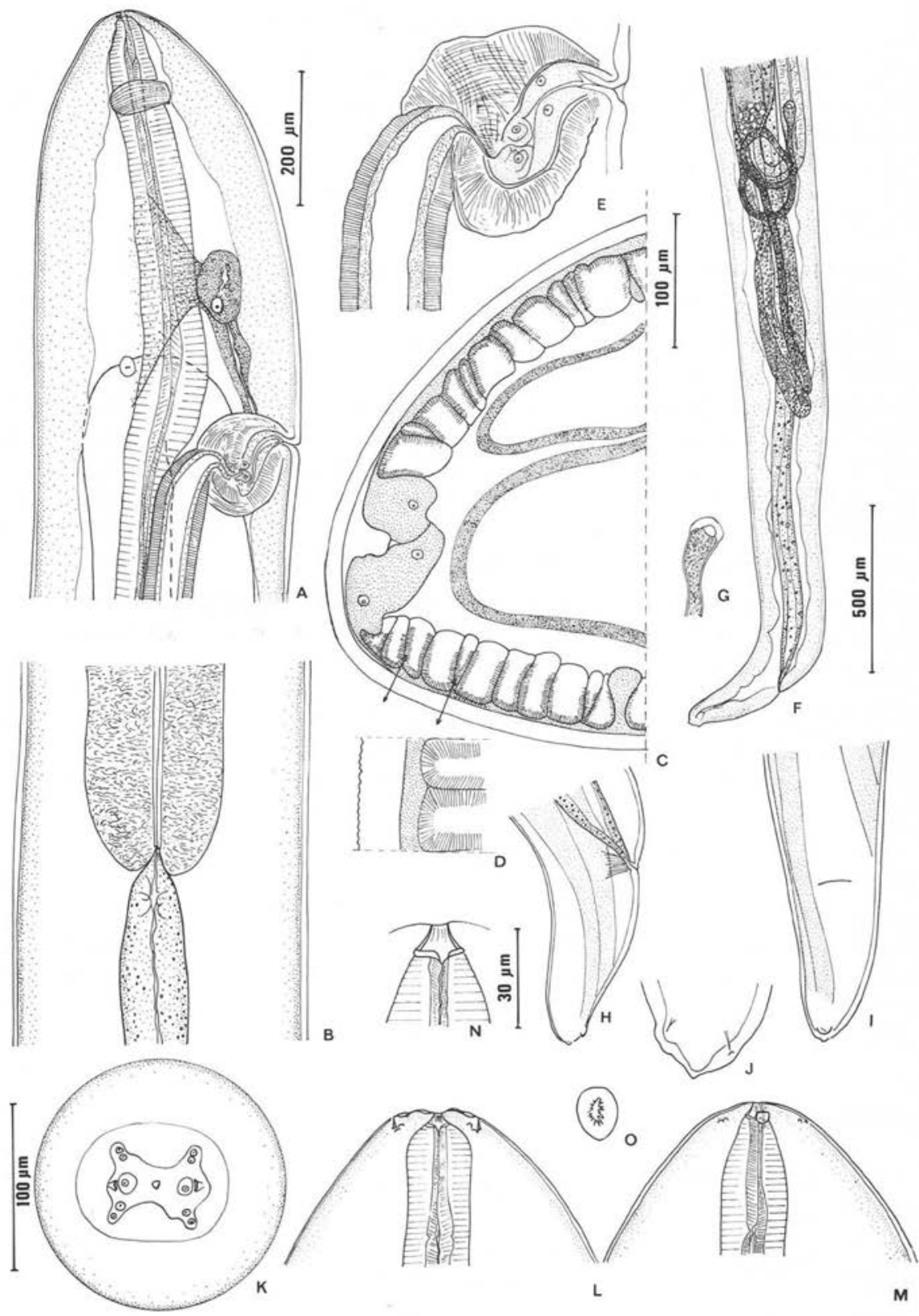

FIG. I. - Chabfilaria jonathani n. gen., n. sp., $Q$; A : région antérieure, vue latérale ; B : jonction œsophage-intestin ; $\mathrm{C}$ : coupe transversale du corps; $\mathrm{D}$ : idem, détail ; $\mathrm{E}$ : vagin vue latérale ; $\mathrm{F}$ : région postérieure, vue latérale; $\mathrm{G}$ : apex d'un des ovaires; $\mathrm{H}$ : queue, vue latérale ; I : idem, vue ventrale; $\mathrm{J}$ : extrémité caudale, vue ventrale; $\mathrm{K}$ : tête, vue apicale; $\mathrm{L}$ et $\mathrm{M}$ : idem, vue médiane et latérale ; $\mathrm{N}$ : cavité buccale ; $\mathrm{O}$ : coupe transversale optique du segment antérieur de la capsule buccale.

(A, B, G, H, I, éch. $200 \mu \mathrm{m} ; \mathrm{C}, \mathrm{E}$, éch. roo $\mu \mathrm{m} ; \mathrm{F}$, éch. $500 \mu \mathrm{m} ; \mathrm{J}, \mathrm{K}, \mathrm{L}, \mathrm{M}$, éch. roo $\mu \mathrm{m}$; D, N, O, éch. $30 \mu \mathrm{m}$ ). 
$10 \mu \mathrm{m}$; œsophage long de $12.300 \mu \mathrm{m}$ avec portion musculaire longue de $500 \mu \mathrm{m}$; vulve à 610 de l'apex; ovaires à 1950 et $29.000 \mu \mathrm{m}$ de l'extrémité caudale; queue longue de $240 \mu \mathrm{m}$.

Mâle (fig. 2).

Area rugosa légèrement dissymétrique formée de bandes transversales de rides cuticulaires longitudinales ( $f i g .2 O$ et $P$ ).

Spicules puissants ( $f i g .2 \mathrm{~K}$ ) ; spicule gauche à manche simple, à pièce intermédiaire développée et à lame complexe ; celle-ci comprend un axe bien sclérifié et, autour, une vésicule cuticulaire striée transversalement ; cette vésicule a son diamètre maximum dans le deuxième tiers de la lame; à ce niveau les stries sont composées de petits reliefs plus ou moins quadrangulaires et alignés ; le spicule gauche se termine en biseau. Le spicule droit est fortement courbé ventralement, très cuticularisé et porte un talon récurrent subterminal. Gubernaculum bien cuticularisé, de forme simple, à section transversale en fer à cheval.

19 à 21 papilles caudales légèrement pédonculées dont la disposition est indiquée sur les figures $2 \mathrm{~F}, G, H, I$ et $J$; les 4 papilles précloacales droites sont décalées vers le cloaque par rapport à celles de la gauche.

Dimensions : $\widehat{\alpha}$ allotype : corps long de $39,9 \mathrm{~mm}$, large de $300 \mu \mathrm{m}$; anneau nerveux et deirides à $100 \mu \mathrm{m}$ et $330 \mu \mathrm{m}$ de l'apex ; œsophage long de $10.260 \mu \mathrm{m}$ avec portion musculaire longue de $630 \mu \mathrm{m}$; queue longue de $190 \mu \mathrm{m}$; spicules gauche et droit longs de 580 et $170 \mu \mathrm{m}$; gubernaculum long de $45 \mu \mathrm{m}$.

Autre $\widehat{\alpha}$ : corps long de $35,5 \mathrm{~mm}$, large de $250 \mu \mathrm{m}$; anneau nerveux, deirides et cellule excrétrice à $110,350 \mu \mathrm{m}$ et $350 \mu \mathrm{m}$ de l'apex ; cavité buccale haute de $8 \mu \mathrm{m}$; œsophage long de $9000 \mu \mathrm{m}$ avec portion musculaire longue de $500 \mu \mathrm{m}$; queue longue de $180 \mu \mathrm{m}$; spicules gauche et droit longs de 470 et $140 \mu \mathrm{m}$; gubernaculum long de $45 \mu \mathrm{m}$; area rugosa commençant à $600 \mu \mathrm{m}$ et finissant à $4.800 \mu \mathrm{m}$ de l'extrémité caudale; rides de l'area rugosa longue de 8-12 $\mu \mathrm{m}$ et bandes de rides espacées de $12-15 \mu \mathrm{m}$.

\section{Microfilaire (fig. 3).}

Gaine présente dépassant la microfilaire aux deux extrémités. Ornementation céphalique de 7 rangées de petits points sur la face opposée au crochet ( fig. $3 C$ à E) ; espace céphalique court ; pores excréteur et anal bien marqués; corps interne court ; queue à extrémité obtuse, parfois un peu renflée, avec 1 ou 2 noyaux terminaux.

Dimensions : microfilaires utérines : corps long de 125 à $138 \mu \mathrm{m}$ et large de 5 à $6 \mu \mathrm{m}$; pour une microfilaire longue de $138 \mu \mathrm{m}$, pore excréteur et corps interne à 48 et $95 \mu \mathrm{m}$ de l'apex ; queue longue de $16 \mu \mathrm{m}$. Microfilaire sanguine : corps long de $138 \mu \mathrm{m}$ et large de $5 \mu \mathrm{m}$; espace céphalique de $2 \mu \mathrm{m}$ de long ; anneau nerveux et pore excréteur à 28 et $45 \mu \mathrm{m}$ de l'apex ; corps interne non coloré ; queue longue de $18 \mu \mathrm{m}$. 


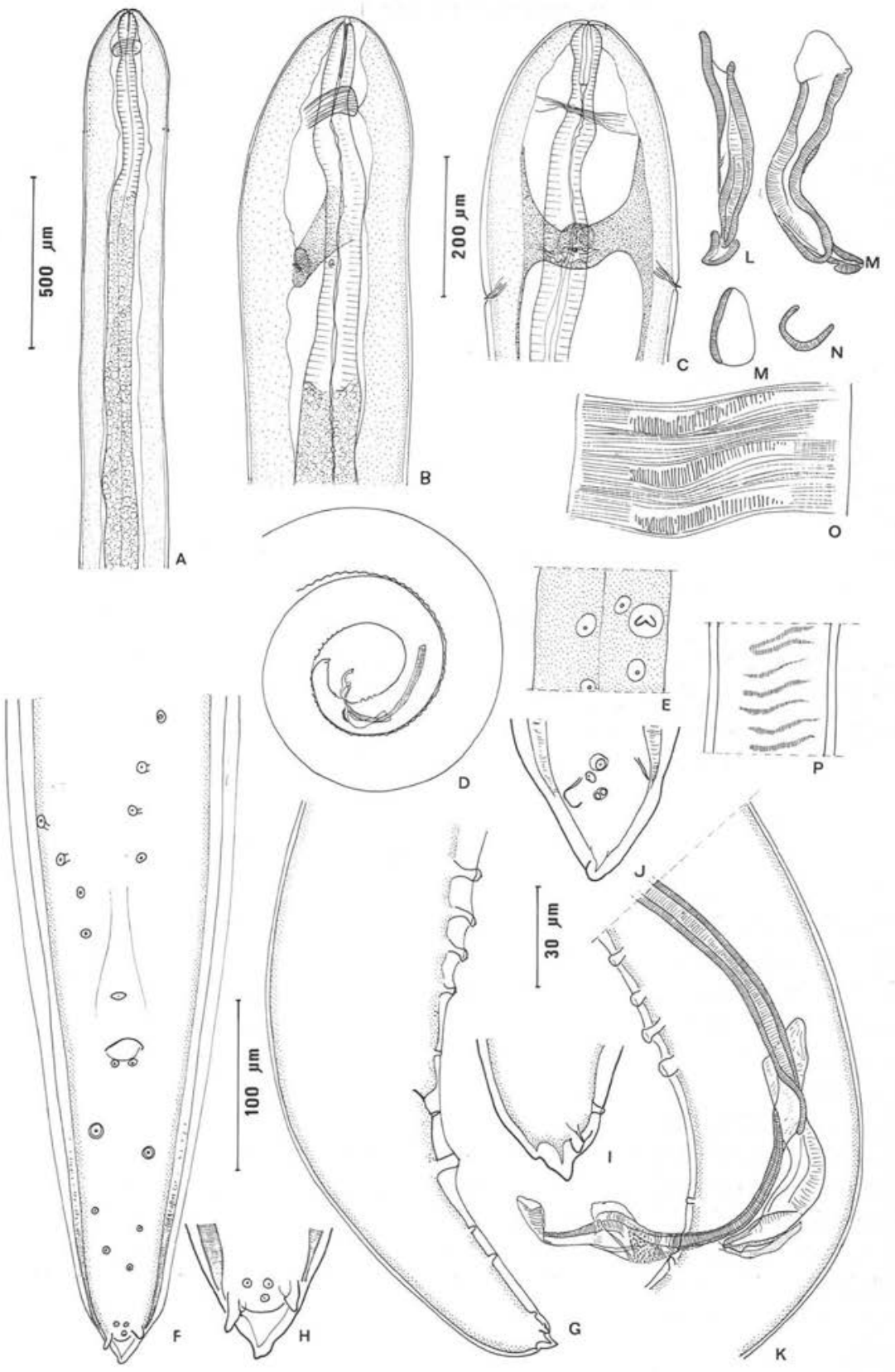

FIG. 2. - Chabfilaria jonathani $\mathrm{n}$. gen., n. sp., $\widehat{0} ; \mathrm{A}$ : région antérieure, vue médiane; $\mathrm{B}$ : région cervicale, vue latérale; $\mathrm{C}:$ idem, vue médiane; $\mathrm{D}$ : région postérieure, vue latérale; $\mathrm{E}$ : deiride et corde latérale; F : région caudale, vue ventrale ; G : idem, vue latérale ; H et I : extrémité caudale, vues ventrale et latérale ; J : idem, vue ventrale, $2^{\mathrm{e}} \hat{\jmath} ; \mathrm{K}$ : spicules et gubernaculum, vue latérale; $\mathrm{L}$ et $\mathrm{M}$ : spicule droit, vues ventrale et latérale; $\mathrm{M}$ et $\mathrm{N}$ : gubernaculum, vues ventrale et coupe optique transversale; $\mathrm{O}$ et $\mathrm{P}$ : area rugosa, vue ventrale.

(A, D, éch. $500 \mu \mathrm{m} ; \mathrm{B}, \mathrm{C}, \mathrm{P}$, éch. $200 \mu \mathrm{m} ; \mathrm{E}, \mathrm{H}, \mathrm{I}, \mathrm{J}$, éch. $30 \mu \mathrm{m}, \mathrm{F}, \mathrm{G}, \mathrm{K}, \mathrm{L}, \mathrm{M}, \mathrm{N}, \mathrm{O}$, éch. $100 \mu \mathrm{m})$. 


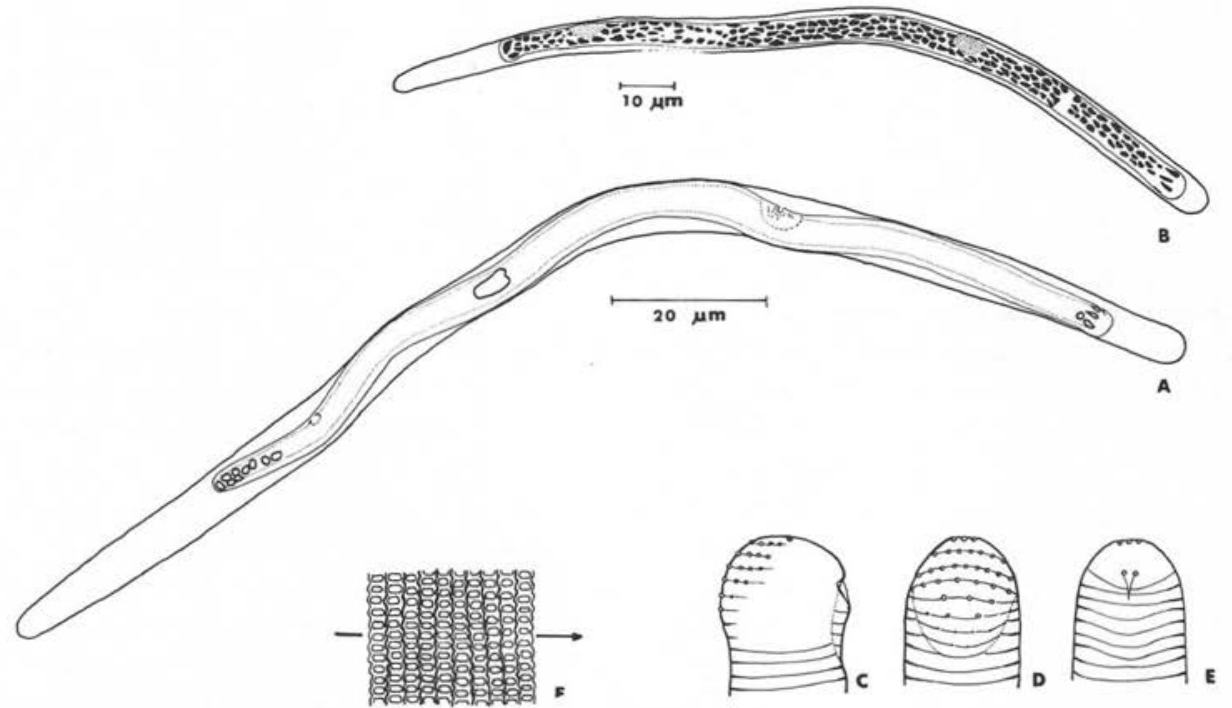

FIG. 3. - Chabfilaria jonathani n. gen., n. sp. ; A à E, microfilaire. A : microfilaire utérine ; $\mathrm{B}$ : microfilaire sanguine colorée à l'hémalun; C : tête, crochet vu de profil ; D : idem, face opposée au crochet ; E : idem, crochet vu de face ; F : ornementation cuticulaire d'une $ᄋ$ (axe longitudinal du corps indiqué par la flèche).

(A : éch. $20 \mu \mathrm{m} ; \mathrm{B}, \mathrm{F}$ : éch. Io $\mu \mathrm{m}$; C à E, à main levée).

\section{Discussion}

Bostrichodera freitaslenti (Yeh, 1957), parasite du Xénarthre Myrmecophaga tridactyla L., est la seule espèce qui soit proche de cette Filaire de Choloepus didactylus.

La tête a la même structure originale avec les 2 volumineuses papilles labiales externes latérales situées près des amphides (ces dernières non représentées par Yeh) et les 2 légers reliefs latéraux situés entre ces papilles et la bouche $(f i g .1 \mathrm{~K}$ et $\mathrm{L}$ et fig. 1 de Yeh).

Le nombre et la disposition des papilles caudales sont similaires : les 4 premières paires nettement précloacales, la paire 5 située juste après le cloaque et subventrale ; la paire 6 volumineuse et plus latérale que la précédente ; les dernières paires étagées sur la moitié postérieure de la queue.

Les autres caractères communs sont le spicule gauche à puissante vésicule cuticulaire, le gubernaculum présent, l'œsophage large, divisé, long d' $1 \mathrm{~cm}$, la queue de la o courbée dorsalement et la forme de la région céphalique (brusquement amincie en avant de l'anneau nerveux).

Toutefois la Filaire du Paresseux est distincte de B. freitaslenti par la vulve plus antérieure (à 500-600 $\mu \mathrm{m}$ de la tête au lieu de $2100-2400 \mu \mathrm{m}$ ), la position asymétrique des papilles précloacales et le rapprochement des 2 dernières paires de papilles caudales. 
Nous avons donc 2 Filaires nettement distinctes mais appartenant à un même genre que nous nommons Chabfilaria n. gen. Ce genre est remarquable par la persistance de nombreux caractères archaïques (papilles labiales externes latérales présentes, grand œsophage divisé, disposition des papilles caudales proche du type Spiruride) et par la morphologie très particulière du spicule gauche.

Yeh, en 1957, avait rapproché la Filaire du Fourmilier d'un parasite du Chameau, D. evansi (Lewis, 1882), espèce type du genre Deraiophoronema Romanovitch, 1916 (Chabaud \& Choquet, 1953).

Une série de caractères communs existe en effet entre cette espèce et freitaslenti mais ce sont tous des caractères primitifs, comme le grand œsophage divisé, les deirides fortes et les papilles caudales non groupées près du cloaque. Les excellentes descriptions données par Boulenger (1924) et par Nagaty (1947) montrent qu'en fait $D$. evansi n'a pas d'affinité particulière avec freitaslenti; la tête, suivie d'un cou, est étirée latéralement et sans papilles labiales externes latérales, la queue de la 우 se termine par 2 mamelons, 1 ventral et 1 dorsal séparés par un sillon transversal, etc.

Chabaud et Bain, en 1976, en se basant sur la description de Yeh qui indique la présence d'ailes caudales chez le $\sigma^{\top}$ de freitaslenti ont proposé de placer cette espèce dans les Dirofilariinae et l'on rattachée au genre Bostrichodera Sandground, 1938 dont l'espèce type est un parasite de Xénarthre, B. becquaerti Sandground, 1938. Ces auteurs placent également dans ce genre une $3^{e}$ Filaire d'Edenté, B. spiralis (Molin, 1860), qui avait déjà été rapprochée de freitaslenti par Yeh (1957) mais qui avait été maintenue par la plupart des auteurs dans le genre Dipetalonema Diesing, 1861. (Cf. Sonin, 1975.)

La Filaire de Choloepus que nous décrivons n'a pas d'ailes caudales chez le $\widehat{\sigma}$; ce fait, joint à d'autres particularités morphologiques communes à cette espèce et à freitaslenti, comme l'œsophage très long, le gubernaculum présent, le spicule gauche à vésicule cuticulaire, séparent en fait nettement ces 2 espèces du petit groupe homogène formé par $B$. becquaerti et $B$. spiralis (cf. redescription de Lent et Freitas, 1942) et également des autres Dirofilariinae.

On constate par contre que plusieurs caractères de Chabfilaria évoquent les Setariinae, parasites d'Ongulés. Ainsi le spicule gauche, avec sa vésicule cuticulaire très particulière, n'existe, à notre connaissance, que chez Setaria equina (Abildgaard, 1789) cf. Yeh, 1959) ; l'œsophage très long, les forts pointes caudales (quand elles existent), la région céphalique épaisse, les deirides bifides (cf. Desset, 1966) sont des particularités qui se rencontrent chez les Setariinae ; la râpe céphalique de la microfilaire est semblable à celle qui a été décrite chez $S$. labiatopapillosa (Alessandrini, 1848) (cf. Bain, 1970) ; enfin, la localisation des 2 Filaires de Xénarthres (muscles de la panse ou cavité abdominale) rappelle celle des Setariinae.

Toutefois Chabfilaria s'oppose aux Setariinae par la morphologie de la tête : ce genre n'a ni anneau péribuccal saillant, ni puissants mammelons latéraux, caractères qui, selon Anderson et Bain (1976) définissent respectivement les 2 genres de Setariinae, Setaria et Papillosetaria ; il n'a que deux très petites bosses céphaliques latérales.

Il est donc possible que Chabfilaria corresponde à une Setaire très primitive 
mais cela reste une hypothèse. On ne peut donc modifier les définitions des sousfamilles pour y inclure ce genre.

Nous préférons le placer dans les Onchocercinae qui groupent déjà des représentants très divers et dont la définition est large (Anderson et Bain, 1976).

Le nouveau genre, Chabfilaria n. gen., dédié au professeur A. G. Chabaud, a la définition suivante :

Chabfilaria n. gen. : Onchocercinae avec papilles labiales externes latérales volumineuses et 2 petites bosses céphaliques latérales; œsophage très long et divisé ; spicule gauche à lame entourée d'une large vésicule cuticulaire; gubernaculum présent ; 9-10 paires de papilles caudales dont 4 paires précloacales et 6 paires disposées le long de la queue; pointes caudales latérales présentes ou absentes. Parasites de Xénarthres.

Espèce type : Chabfilaria jonathani n. sp., parasite de Choloepus didactylus L. (Bradypodidae) en Guyane française.

Autre espèce : Chabfilaria freitaslenti (Yeh, 1957) n. comb., parasite de Myrmecophaga tridactyla L. (Myrmecophagidae), en Guyane anglaise.

\section{Conclusion}

On connaît maintenant 11 espèces de Filaires parasites de Xénarthres. - 6 Dirofilariinae : 4 Dirofilaria sans grandes particularités morphologiques (cf. Eberhard, 1978), mais aussi 2 Bostrichodera qui peuvent être considérés comme les plus primitifs des Dirofilaria parasites de Mammifères.

- 5 Onchocercinae : 2 Orihelia, 1 Dasypafilaria; ce dernier parait une forme spécialisée d'Orihelia; Orihelia lui-même est interprété comme un genre très primitif (Bain, Baker et Chabaud, 1982), peu éloigné de Skrjabinofilaria et constituant donc un des groupes les plus primitifs des Onchocercinae.

Les deux Chabfilaria enfin présentent également une morphologie très primitive et correspondent peut-être à des ancêtres des Setariinae.

Les trois grands groupes de Filaires actuellement diversifiés chez les Mammifères, c'est-à-dire les Dirofilariinae, les Onchocercinae et les Setariinae, auraient des formes primitives chez les Xénarthres.

On peut imaginer que la zone néotropicale du début du Tertiaire, peuplée de Marsupiaux, de Xénarthres et de Protongulés, a joué un rôle important dans l'origine des Filaires de Mammifères.

\section{BIBLIOGRAPHIE}

Anderson R. C., BaIn O. : CIH Keys to the Nematode Parasites of Vertebrates. No 3 . Keys to genera of the Order Spirurida. Part 3. Diplotriaenoidea, Aproctoidea and Filarioidea. Edit. Anderson, Chabaud, Willmott, Commonwealth Institute of Helminthology, St. Albans (Herts.), England, 1976, 59-116.

Barn O. : Morphologie larvaire de Setaria labiatopapillosa (Nematoda, Filarioidea) chez Aedes aegypti. Ann. Parasitol. Hum. Comp., 1970, 45, 431-439. 
Bain O., Baker M., Сhabaud A. G. : Nouvelles données sur la lignée Dipetalonema (Filarioidea, Nematoda). Ann. Parasitol. Hum. Comp., 1982, 57, 593-620.

Boulenger C. L. : The filariid of the camel, Acanthocheilonema evansi (Lewis) Parasitology, I924, I 6, 4I9-423.

Chabaud A. G., Bain O. : La lignée Dipetalonema. Nouvel essai de classification. Ann. Parasitol. Hum. Comp, 1976, s1, 365-397.

Chabaud A. G., Choquet M. T. : Nouvel essai de classification des Filaires (Superfamille des Filarioidea). Ann. Parasitol. Hum. Comp., 1953, 28, I72-192.

Desset M.-C. : Contribution à la systématique des Filaires du genre Setaria; valeur des deirides. Mém. Mus. natn. Hist. nat., nouv. Sér., Sér. A, Zool., 1966, 39, 257-285.

EBERHARD M. L. : Dirofilaria macrodemos and D. panamensis spp. n. (Nematoda : Filarioidea) from Central and South American Sloths. J. Parasitol., 1978, 64, 198-203.

Lent H., Freitas J. F. : Sobre Dipetalonema spiralis (Molin, I860) (Nematoda, Filarioidea). Mem. Inst. Oswaldo Cruz, 1942, 37, 383-389.

Nagaty H. F. : Dipetalonema evansi (Lewis, I882) and its microfilaria from Camelus dromedarius. Parasitology, 1947, 38, 86-92.

Sandground J. H. : On three species of Filariid Nematodes from Sloths. Livro Jubilar Prof. Travassos, Rio de Janeiro, Brasil, III, $1938,42 \mathrm{I}-428$.

SonIN M. D. : (Filaires des animaux et de 1'homme et maladies causées par elles. 3. Filariidae et Onchocercidae). Osnovi Nematodologii, Akad. Nauk SSR, Moscou, 1975, 24, 396 p.

YEH, L. S. : On a filarial parasite Deraiophoronema freitaslenti $\mathrm{n}$. sp. from the giant anteater, Myrmecophaga tridactyla from British Guiana, and a proposed reclassification of Dipetalonema and related genera. Parasitology, 1957, 47, 196-205.

YEH, L. S. : A revision of the nematode genus Setaria Viborg, I 795, its host-parasite relationship, speciation and evolution. J. Helminthol., 1959, 33, I-I86. 